Mathématiques et sciences humaines
Mathematics and social sciences

$199 \mid 2012$

Psychologie et mathématiques

\title{
Power vs. logarithmic model of Fitts' law: a mathematical analysis
}

Modèle de puissance vs. logarithmique de la loi de Fitts : une analyse

mathématique

Olivier Rioul and Yves Guiard

\section{CpenEdition}

\section{Journals}

Electronic version

URL: http://journals.openedition.org/msh/12317

DOI: $10.4000 / \mathrm{msh} .12317$

ISSN: 1950-6821

Publisher

Centre d'analyse et de mathématique sociales de l'EHESS

Printed version

Date of publication: 15 September 2012

Number of pages: 85-96

ISSN: 0987-6936

\section{Electronic reference}

Olivier Rioul and Yves Guiard, "Power vs. logarithmic model of Fitts' law: a mathematical analysis », Mathématiques et sciences humaines [Online], 199 | 2012, Online since 04 December 2012, connection on 23 July 2020. URL : http://journals.openedition.org/msh/12317 ; DOI : https://doi.org/10.4000/ msh. 12317 


\title{
POWER VS. LOGARITHMIC MODEL OF FITTS' LAW: A MATHEMATICAL ANALYSIS ${ }^{1}$
}

\author{
Olivier RIOUL ${ }^{2}$, Yves GUIARD ${ }^{2}$
}

\begin{abstract}
RÉSUMÉ - Modèle de puissance vs. logarithmique de la loi de Fitts : une analyse mathématique. Après bientôt soixante années d'études, il reste toujours à déterminer si la loi de Fitts, un modèle célèbre du mouvement de pointage humain, est une loi logarithmique ou de puissance. Dans deux articles abondamment cités, Meyer $\mathscr{E}$ al. ont avancé l'idée que le modèle de puissance qu'ils ont déduit de leur théorie stochastique des sous-mouvements optimisés englobe le modèle logarithmique comme un cas limite atteint lorsque le nombre de sous-mouvements devient grand. Reconsidérant la théorie des sous-mouvements de Meyer et al., nous montrons que cette proposition est mathématiquement inexacte. La théorie de Meyer et al. implique en réalité un modèle quasi-logarithmique plutôt que de puissance, le premier n'étant pas équivalent au second. Une pleine conscience que les deux classes possibles de description mathématique de la loi de Fitts ne sont pas équivalentes nous semble de nature à stimuler la recherche expérimentale dans ce domaine.
\end{abstract}

MOTS-CLÉS - Loi de Fitts, Équations fonctionnelles, Modèles mathématiques en psychologie, Pointage, Mouvement rapide orienté vers une cible

SUMMARY - Whether Fitts' law, a well-known model of human pointing movement, is a logarithmic law or a power law has remained unclear so far. In two widely cited papers, Meyer 83 al. have claimed that the power model they derived from their celebrated stochastic optimizedsubmovement theory encompasses the logarithmic model as a limiting case, when the number of submovements grows large. We review the Meyer 85 al. submovement theory and show that this claim is questionable mathematically. Our analysis reveals that Meyer $\& 6$ al.'s theory implies in fact a quasi-logarithmic, rather than quasi-power model, the two models not being equivalent. Awareness that the two classes of candidate mathematical descriptions of Fitts' law are not equivalent should stimulate experimental research in the field.

KEYWORDS - Fitts' law, Functional equations, Mathematical models in psychology, Pointing, Simple rapid aimed movement

\section{INTRODUCTION}

Fitts' law [Fitts, 1954; Fitts \& Peterson, 1964; Plamondon \& Alimi, 1997] is a well-known empirical regularity which predicts the average movement time $T$ it takes people, under time pressure, to reach a target of width $W$ located at distance $D$

\footnotetext{
${ }^{1}$ Part of this paper was presented at the 2011 Meeting of the European Mathematical Psychology Group [Rioul \& Guiard, to appear].

${ }^{2}$ Télécom ParisTech, CNRS LTCI, 46 rue Barrault 75634 Paris Cedex 13, (olivier.rioul, yves.guiard)@telecom-paristech.fr
} 
(see Figure 1) with some pointer. This model has proven useful in several fields of applied psychology such as Human-Computer Interaction and Ergonomics.

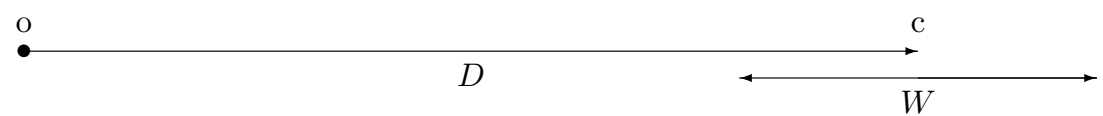

FIGURE 1. One-dimensional single-shot movement paradigm settled by Fitts [Fitts \& Peterson, 1964]. Starting at origin $o$, the pointer must be moved rapidly to target center $c$, the target being displayed as a tolerance interval of width $W$.

The mathematical formulation of Fitts' law is as follows: movement time $T$ is linearly dependent on an index of difficulty [Fitts \& Peterson, 1964] ID, which in turn is a strictly increasing function $f$ of the relative distance $D / W$. Thus

$$
T=a+b \cdot f(D / W),
$$

where $a$ and $b$ are adjustable constants. The question is to know what the shape of $f$ is. Many different formulations have been proposed for $f$ in the literature over the past fifty years, none of which has been empirically disproved. Perhaps the most favorably received are:

$$
\mathrm{ID}=f(D / W)= \begin{cases}\log _{2} \frac{2 D}{W} & \text { Fitts [1954] } \\ \log _{2}\left(0.5+\frac{D}{W}\right) & \text { Welford [1960] } \\ \log _{2}\left(1+\frac{D}{W}\right) & \text { McKenzie [1989] } \\ \sqrt{\frac{D}{W}} & \text { Meyer et al. }[1988] \\ \left(\frac{D}{W}\right)^{1 / n} & \text { Meyer et al. }[1990]^{3}\end{cases}
$$

In particular, whether Fitts' law is a logarithmic (1a)-(1c) or a power law (1d)-(1e) has remained unclear so far. One plausible explanation is that the practical range of relative distances $D / W$ that can be actually investigated in the laboratory is rather narrow [Guiard \& Olafsdottir, 2011]:

$$
3 \lesssim \frac{D}{W} \lesssim 33
$$

This is because for $D / W \ll 3$ experimenters have a floor effect on $T$ (speed saturation) while for $D / W \gg 33$ the error rate tends to explode (accuracy saturation). Within the range $[3,33]$, the curves (1a)-(1e) are indeed similar (see Figure 2), making it difficult to decide empirically between the logarithmic and the power model.

\footnotetext{
${ }^{3}$ Here $n$ is an integer representing the maximum "number of submovements" [Meyer et al., 1990] and (1d) corresponds to $n=2$. The paternity of the power law was later claimed by Kvålseth [1993], who showed that the formulation $T=a(D / W)^{b}$ achieves higher fits to Fitts' data [Kvålseth, 1980].
} 


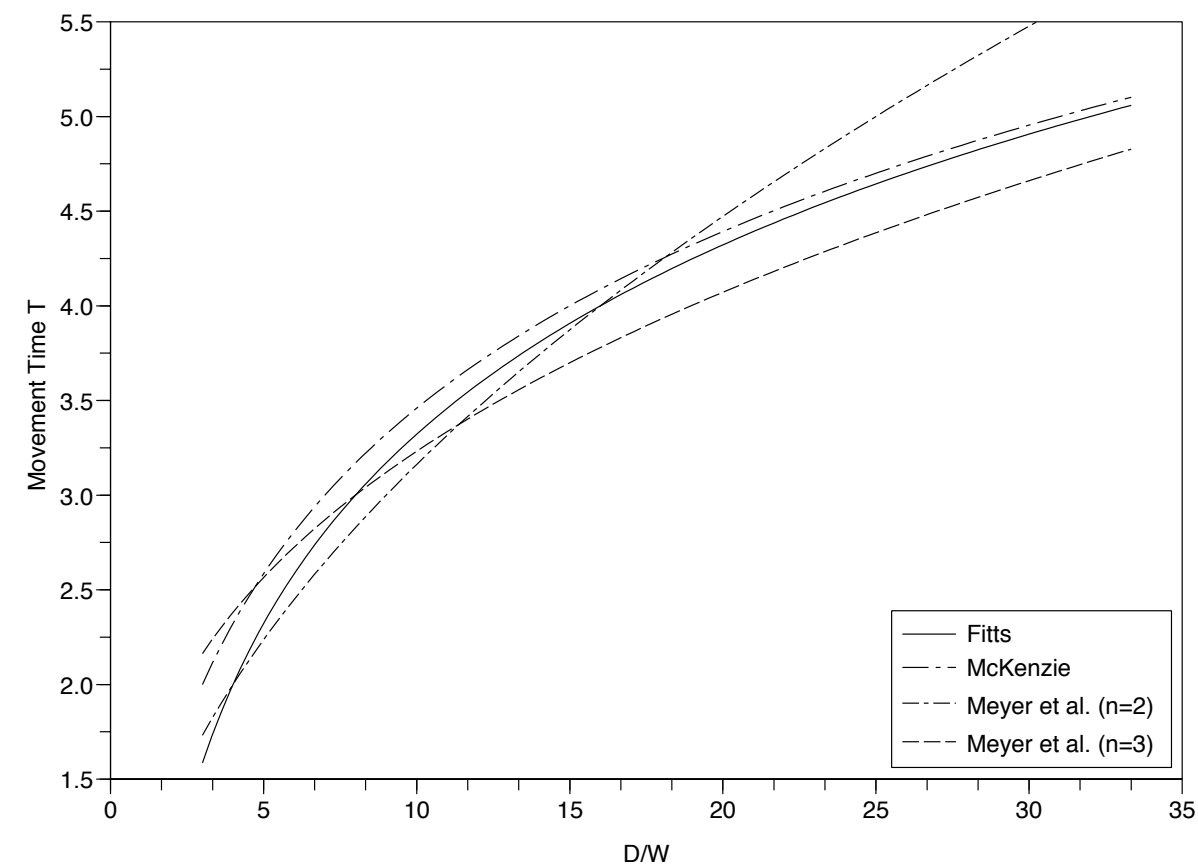

Figure 2. Movement time $T$ vs. $D / W$ for the four formulations of (1) in the range (2). Eq. (1d) yields a square-root law while Eq. (1e) is plotted with $n=3$ (cube-root law).

Such irresolution would not be a problem if mathematical models were just black boxes serving to deliver numerical simulations of empirical data - that minimal job is done about equally well by the logarithmic and the power model of Fitts' law. Thus, from a practical perspective, one could always support the claim that one mathematical model or another accurately approximates the data.

But our concern is more theoretical. In general Fitts' law researchers do care about the internal structure of their mathematical formulas, which they typically take as models of the law they want to understand. Ideally a mathematical model, as distinct from a blind numerical simulation, is a short statement whose variables, parameters, and relations explicitly map onto quantities and relations of some substantive theory. For example, in $(1 \mathrm{c})$ the term $\log _{2}(1+D / W)$ is meant to represent a measure of Shannon's information capacity [Shannon, 1948] and so the logarithmic form of that model is a deliberate, theoretically-driven option. Likewise, it is an elaborate theoretical analysis that led Meyer et al. [1990] to put forth the power equation of $(1 \mathrm{e})$, in which the parameter $n$ is supposed to represent the maximum number of submovements a participant is willing to make.

Despite the similarity of predictions within the range of interest (2), the logarithmic vs. power form of Fitts' law is indeed a theoretically-important empirical issue, and so there is reason to be concerned by the lack of consensus. To paraphrase Platt [1964, p. 351], "a failure to agree for 60 years is public advertisement of a failure to disprove."

In two widely cited papers Meyer et al. [Meyer et al., 1988, 1990] argued that $a+b \cdot(D / W)^{1 / n}$ tends to $a^{\prime}+b^{\prime} \cdot \log (D / W)$ as the maximum number $n$ of submovements tends to infinity. This suggests that there is no real logarithmic vs. power issue 
about Fitts' law: the power model they derived from their celebrated stochastic optimized-submovement theory would encompass the logarithmic model as a limiting case.

It turns out, however, that this claim is questionable. We propose a detailed analysis to show that Meyer et al.'s model does not predict a genuine power law but rather some quasi-logarithmic law, the two classes of candidate mathematical descriptions of Fitts' law being not equivalent.

This paper is organized as follows. In Section 2, after reviewing some mathematical derivations of Fitts' law based on submovement models, we restate Meyer et al.'s claim and disprove it. We then review the stochastic optimized-submovement model in Section 3 to explain analytically why the power formulation fails as a mathematical description of Fitts' law for multiple submovements. Section 4 concludes.

\section{PREVIOUS WORK AND A DISPROOF}

We begin with a short review of two major mathematical derivations of Fitts' law. Both assume that the pointing movement toward the target is composed of a sequence of $n$ submovements.

\subsection{DETERMINISTIC ITERATIVE-CORRECTIONS MODEL}

The deterministic iterative-corrections model was originally formulated by Crossman and Goodeve [1963]. The model assumes that each submovement (i) requires a constant time $\Delta T$ to be executed and (ii) covers a constant fraction $\lambda<1$ of the remaining distance to the center of the target. The target is reached if that remaining distance is equal to half the target width $W / 2$.

It follows that the required time $T=n \Delta T$ to reach the target is such that $\lambda^{n} D=W / 2$. This is easily seen to imply

$$
T=c \log _{2} \frac{2 D}{W}
$$

where $c=\Delta T \log _{2}(1 / \lambda)$, that is, a logarithmic formulation of the form (1a).

This model, while being very simple, has long been abandoned because it relies on rather strong assumptions (i,ii) that have been empirically observed to be wrong [Plamondon \& Alimi, 1997, § 2.1.2]. Also, one fatal objection is that the model ignores submovement endpoint variability, and thus fails to predict any movement endpoint spread and to accommodate the possibility of target misses, most notably overshoots [Meyer et al., 1988, 1990; Plamondon \& Alimi, 1997].

\subsection{STOCHASTIC OPTIMIZED-SUBMOVEMENT MODEL}

The stochastic optimized-submovement model proposed by Meyer et al. [1988] assumes a random spread of submovement endpoints. The authors follow Woodworth's 1899 suggestion [Woodworth, 1899] that the movement involves $n=2$ successive phases $^{4}$ : There is an initial 'ballistic' submovement for which the endpoint spread is

\footnotetext{
${ }^{4}$ The model was also extended to multiple submovements (arbitrary large $n$ ): see Section 3.
} 
proportional to velocity, and whose duration is given by [Smith, 1988]

$$
T_{i}=\frac{D / W-1 / 2}{s}
$$

where $s$ is some spread parameter ${ }^{5}$. Let $\Delta$ be the resulting distance to the target center; $\Delta$ is modeled by a stochastic (random) variable - following, e.g., a Gaussian distribution - whose standard deviation is proportional to $s$.

Next, if the target is not reached yet $(|\Delta|>W / 2)$, a secondary submovement occurs. The total average time (within a constant multiplicative factor) is the sum:

$$
T=\min _{s}\left\{\frac{D / W-1 / 2}{s}+\mathbf{E}_{|\Delta|>W / 2}\left(\frac{|\Delta|}{W}\right)\right\}
$$

where the expectation $\mathbf{E}$ is with respect to $\Delta$ 's distribution over the region $|\Delta|>W / 2$. The optimization takes the form of a minimization over $s$, the only free variable left [Meyer et al., 1988, appendix].

Meyer et al. found that the solution is proportional to

$$
T \propto \frac{2 \theta \sqrt{D / W}-\sqrt{W / D}}{\theta \sqrt{\theta-W / D}}
$$

where $\theta$ is in fact a complicated function of $D / W$ such that $\theta=k \exp \frac{1}{2(\theta D / W-1)}$ and is determined iteratively by a fixed point theorem [Meyer et al., 1988, appendix] for a given value of proportionality factor $k$. The result is well aproximated by a square-root law of the form (1d) (see Figure 3).

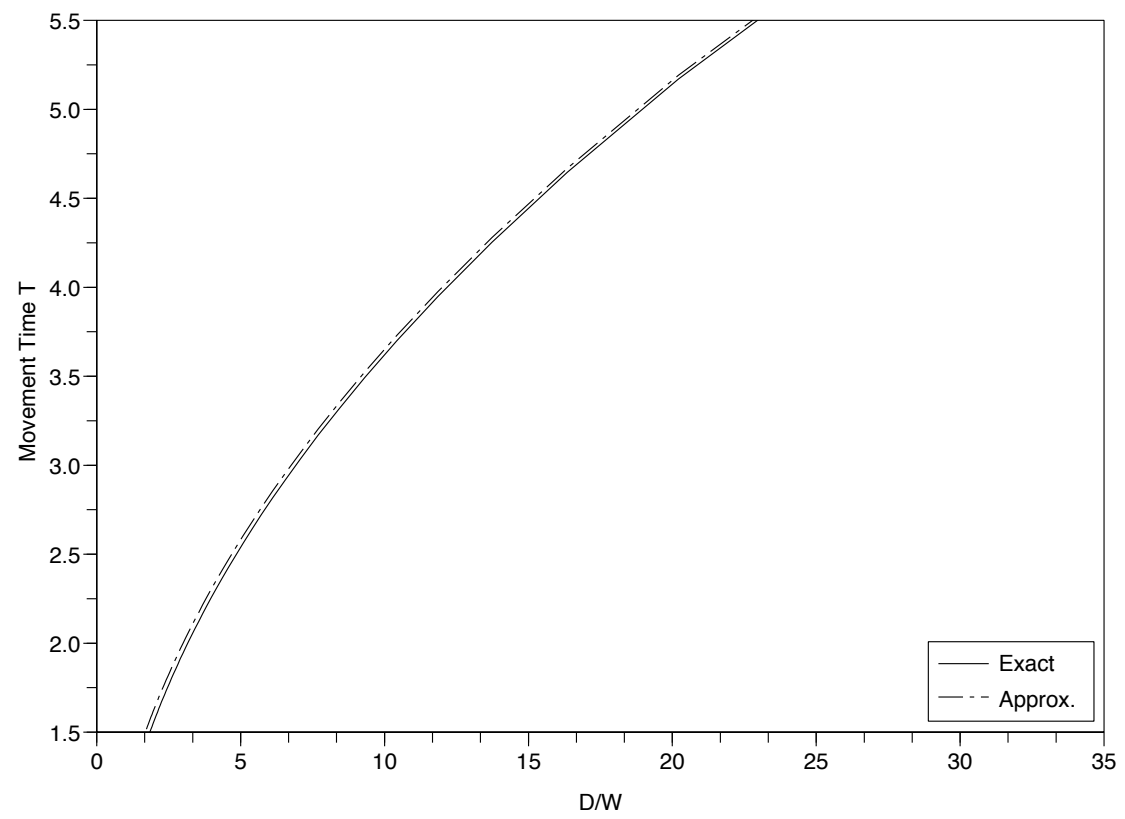

FIGURE 3. Movement time $T$ vs. $D / W$ for the exact solution (4) (solid) and corresponding square-root approximation (dashed).

\footnotetext{
${ }^{5}$ Following [Smith, 1988], a term $1 / 2$ is introduced so that $T=0$ when the origin already lies in the target.
} 
The stochastic optimized-submovement model can be easily extended to multiple submovements (see subsection 3.1 below). Meyer et al. [1990] claimed that for $n$ submovements, the model yields a solution that can likewise be approximated by a $n$th root law of the form (1e).

\subsection{MEYER et al.'s [1988, 1990] CLAIM FOR MULTIPLE SUBMOVEMENTS: A DISPROOF}

The claim is as follows (see Figure 4):

Mathematically, $\log _{2}(D / W)$ is equivalent to the limiting case of a power function $(D / W)^{x}$ of $D / W$ whose positive exponent $x$ tends to zero [Meyer et al., 1988, footnote 13].

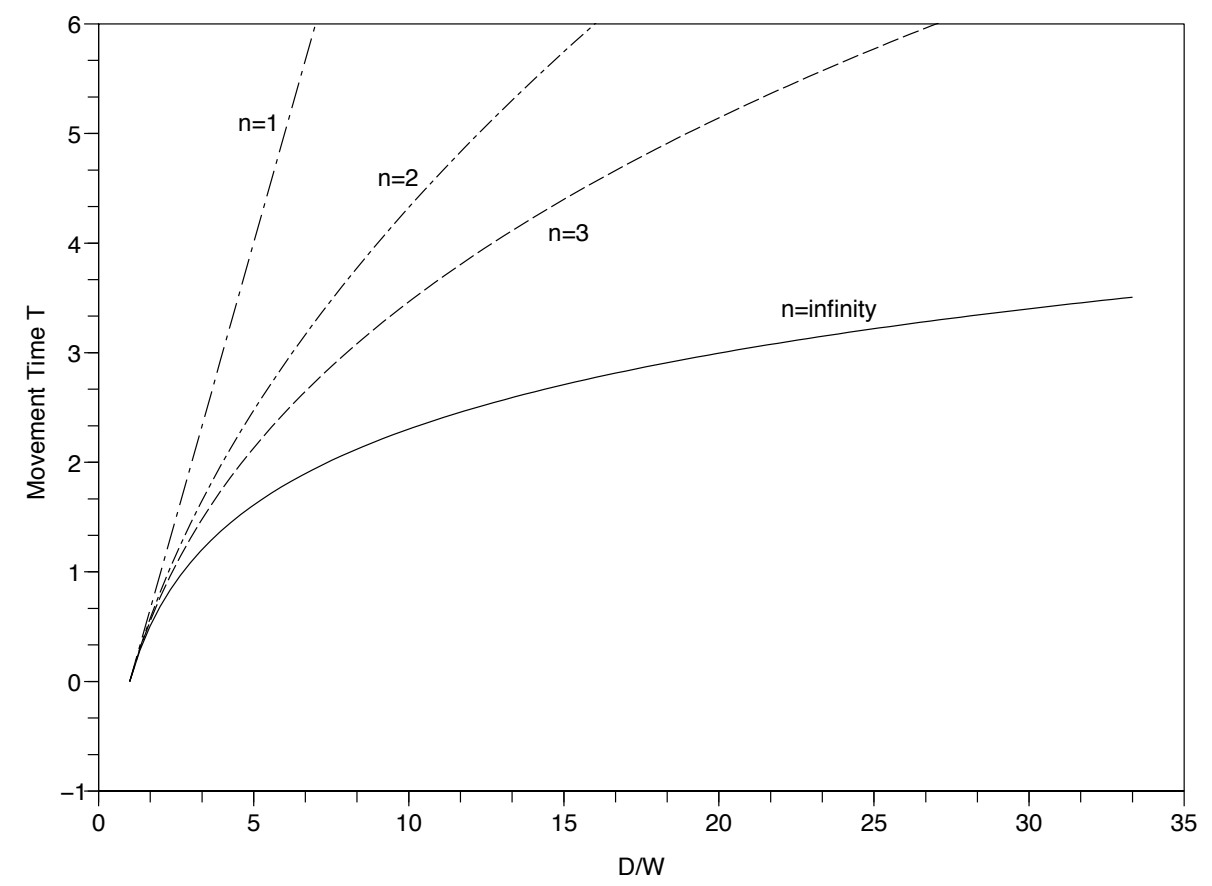

FIGURE 4. A remake of Meyer et al.'s [1990] original Figure 6.13 giving their hypothetical $T$ vs. $D / W$. They claim that as $n$ grows large, their power relation "approaches a logarithmic function, paralleling Fitts' Law' [Meyer et al., 1990].

Meyer et al.'s widely cited papers $[1988,1990]^{6}$ have convinced the Fitts' law research community that their power law encompasses the logarithmic law at the extreme case $n=\infty$ (see e.g. Wobbrock et al. [2008, Eq. (18)] for a recent account).

It turns out, however, that the above claim is mathematically questionable. For any fixed value of $D / W>0$, when the exponent $x=1 / n$ tends to zero,

$$
\sqrt[n]{D / W}=(D / W)^{1 / n}=\exp \left(\frac{1}{n} \log _{e}(D / W)\right)
$$

\footnotetext{
${ }^{6}$ At the time of this writing, [Meyer et al., 1988, 1990] received 744 and 225 citations, respectively. Source: Google Scholar.
} 
has first order approximation $1+\frac{1}{n} \log _{e}(D / W)$ but tends to $\exp 0=1$ as $n \rightarrow+\infty$. Hence the limit is a 'constant law', which is not even a strictly increasing function of $D / W$ and is, therefore, inadequate as a model for Fitts' law ${ }^{7}$. This was apparently first noticed by one of us [Guiard et al., 2001, footnote 6].

Since we took (1e) for granted, our disproof raises the question of the actual validity of the power law. Also, since a square-root or cube-root function is certainly not constant, it makes the dependence on $n$ questionable: different $n$th root laws $(1 \mathrm{e})$ appear different for different values of $n$, including large ones. In the next section, we attempt to solve these problems.

\section{DETAILED ANALYSIS FOR $n$ SUBMOVEMENTS}

We now review the submovement theory of Meyer et al. for multiple submovements [Meyer et al., 1990; Smith, 1988] to explain analytically why the $n$ th-root model fails.

\subsection{DERIVATION}

Let $T=f_{n}(D / W)$ be the average movement time required to reach the target after $n$ submovements. After the initial 'ballistic' submovement, there remain $n-1$ submovements to reach the target located at random distance $|\Delta|$. The stochastic optimized-submovement model (3) then predicts:

$$
f_{n}\left(\frac{D}{W}\right)=\min _{s}\left\{\frac{D / W-1 / 2}{s}+\mathbf{E}_{|\Delta|>W / 2} f_{n-1}\left(\frac{|\Delta|}{W}\right)\right\}
$$

for any $n>1$. To simplify the notation let $\delta$ denote any value of $D / W$ and let $t$ denote any time value. To simplify the calculations we follow Smith's [1988] assumption that $\Delta$ 's distribution is uniform in the interval $(-W s / 2, W s / 2)^{8}$ :

$$
f_{n}(\delta)=\min _{s}\left\{\frac{\delta-1 / 2}{s}+\frac{2}{s} \int_{1 / 2}^{s / 2} f_{n-1}(\delta) \mathrm{d} \delta\right\}
$$

Note that the factor 2 accounts for undershoots as well as overshoots. It is easily seen by induction that $f_{n}$ is well defined, regular (indefinitely continously differentiable) and strictly increasing in the range $\delta>1 / 2$. We can, therefore, define its inverse function $\delta=g_{n}(t)$. It turns out [Smith, 1988] that the determination of relative distance vs. time (that is, of $g_{n}$ ) is easier than the direct determination of $f_{n}$, that is, of time vs. relative distance as in the classical formulation of Fitts' law.

\footnotetext{
${ }^{7}$ The illustration made in [Meyer et al., 1990, Figure 6.13] (see Figure 4) would suggest a weaker claim: there exist sequences $\left(a_{n}\right),\left(b_{n}\right)$ of real numbers with $b_{n}>0$ and constants $a$ and $b>0$ such that $\lim _{n \rightarrow \infty}\left(a_{n}+b_{n}\left(\frac{D}{W}\right)^{1 / n}\right)=a+b \cdot \log _{e}\left(\frac{D}{W}\right)$. This is in fact possible by setting $b_{n}=a-a_{n}=n \cdot b$ for all $n$, so that the curves all intersect at $D / W=1$. During the review process, we became aware that a similar statement was put forth by Kvålseth [1993]. This implies, however, that $\left(a_{n}\right),\left(b_{n}\right)$ are unbounded sequences so that $T=a_{n}+b_{n}\left(\frac{D}{W}\right)^{1 / n}$ is not a genuine power model: Section 3 offers an explanation.

${ }^{8}$ Calculations run similarly for other distributions (e.g., Gaussian), with just more intricate results.
} 
We now derive a simple proof leading to Smith's solution to (6). Making the first derivative of (6) vanish, the optimal $s=s(\delta)$ satisfies

$$
-\frac{\delta-1 / 2}{s^{2}}-\frac{2}{s^{2}} \int_{1 / 2}^{s / 2} f_{n-1}(\delta) \mathrm{d} \delta+\frac{1}{s} f_{n-1}\left(\frac{s}{2}\right)=0,
$$

which boils down to the condition

$$
f_{n}(\delta)=f_{n-1}(s / 2)
$$

Now, by inverting arguments according to $\delta=g_{n}(t)$, (6) can be rewritten as

$$
\begin{aligned}
& t=\frac{g_{n}(t)-1 / 2}{s}+\frac{2}{s} \int_{g_{n-1}(0)}^{g_{n-1}(t)} f_{n-1}(\delta) \mathrm{d} \delta \\
& t=\frac{g_{n}(t)-1 / 2}{s}+\frac{2}{s}\left(\frac{s}{2} \cdot t-\int_{0}^{t} g_{n-1}(\tau) \mathrm{d} \tau\right)
\end{aligned}
$$

where we have used (7) in the form $g_{n-1}(t)=s / 2$ in (8) and the inverse function integration theorem (see Figure 5) in (9). After subtracting $t$ on both sides of (9)

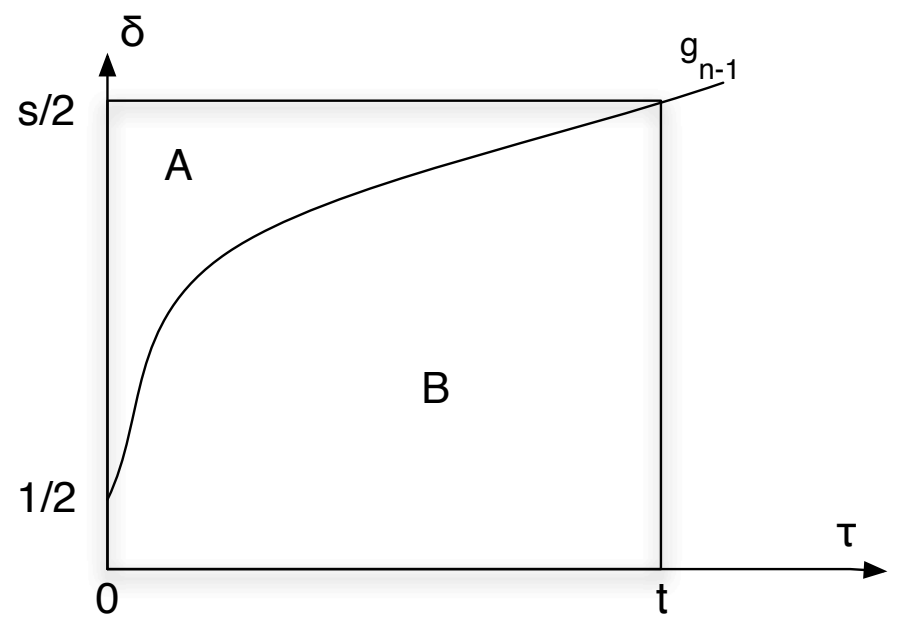

FIGURE 5. The inverse function integration theorem states that the sum of the two areas $A=\int_{1 / 2}^{s / 2} f_{n-1}(\delta) \mathrm{d} \delta$ and $B=\int_{0}^{t} g_{n-1}(\tau) \mathrm{d} \tau$ is equal to $A+B=\frac{s}{2} \cdot t$, the area of the rectangle.

we end up with a simple recursion relation

$$
g_{n}(t)=\frac{1}{2}+2 \int_{0}^{t} g_{n-1}(\tau) \mathrm{d} \tau
$$

which is easily solved by induction. One finds:

$$
g_{n}(t)=\frac{1}{2}+\frac{1}{2}(2 t)+\frac{1}{2} \frac{(2 t)^{2}}{2}+\cdots+\frac{1}{2} \frac{(2 t)^{n}}{n !}=\frac{E_{n}(2 t)}{2},
$$


where

$$
E_{n}(t)=1+t+\frac{t^{2}}{2 !}+\cdots+\frac{t^{n}}{n !}
$$

denotes the $n$th partial sum of the Taylor series of the exponential exp $(t)$. Denoting its inverse function by $L_{n}=E_{n}^{-1}$, one obtains the following law:

$$
T=\frac{1}{2} L_{n}\left(2 \frac{D}{W}\right)
$$

\subsection{CLOSED-FORM EXPRESSIONS}

For each $n$, the exact solution (12) is obtained by solving an $n$th degree equation of the form $E_{n}(t)=0$. We can compute $L_{n}$ for the first few values of $n$.

For $n=2$ one immediately obtains

$$
L_{2}(x)=\sqrt{2 x-1}-1
$$

which implies

$$
T=\sqrt{D / W-1 / 4}-1 / 2 .
$$

Thus one recovers the quasi square-root law of Meyer et al. [1988].

For $n=3$ one obtains

$$
L_{3}(x)=\sqrt[3]{3 x+\sqrt{9 x^{2}-6 x+2}-1}-\frac{1}{\sqrt[3]{3 x+\sqrt{9 x^{2}-6 x+2}-1}}-1,
$$

which is a rather complex "quasi cube-root law:"

$$
T=\frac{1}{2} \sqrt[3]{6 \frac{D}{W}+\sqrt{36\left(\frac{D}{W}\right)^{2}-12 \frac{D}{W}+2}-1}-\frac{1}{2 \sqrt[3]{6 \frac{D}{W}+\sqrt{36\left(\frac{D}{W}\right)^{2}-12 \frac{D}{W}+2}-1}}-\frac{1}{2} .
$$

The case $n=4$ :

$$
\begin{aligned}
& L_{4}(x)=\frac{1}{\sqrt{\frac{\sqrt[3]{192 x+32 \sqrt{32 x^{3}-12 x^{2}+12 x-3}-32}-\sqrt[3]{192 x+32 \sqrt{32 x^{3}-12 x^{2}+12 x-3}-32}}{\sqrt{-\frac{32 x-\sqrt[3]{\left(192 x+32 \sqrt{32 x^{3}-12 x^{2}+12 x-3}-32\right)^{2}}+4 \sqrt[3]{192 x+32 \sqrt{32 x^{3}-12 x^{2}+12 x-3}-32}-16}{\sqrt[3]{192 x+32 \sqrt{32 x^{3}-12 x^{2}+12 x-3}-32}}}}-8}}
\end{aligned}
$$

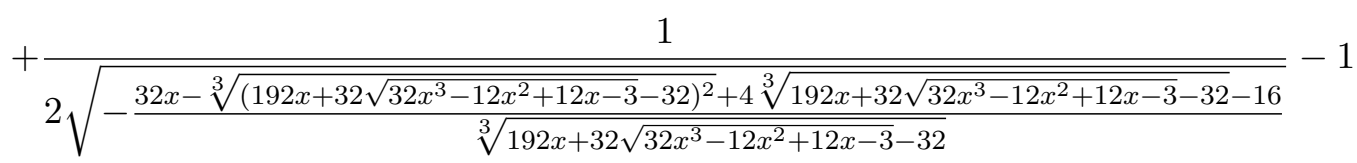

cannot be easily identified with a "fourth-root law" and is probably too intricate to be of any practical use.

For $n>4$ the equation $E_{n}(t)=0$ is not even solvable by radicals and so there exists no closed-form expression. It follows that for large $n,(12)$ cannot be identified with an $n$ th-root (power) formulation of Fitts' law. Thus, the conclusion of Meyer et al. [1988, 1990] that Fitts' logarithmic formulation arises out of a power law with an infinite number of submovements is doubtful. The next section confirms that doubt. 


\subsection{QUASI-POWER VS. QUASI-EXPONENTIAL LAWS}

In our derivation, the case $|\Delta| \leq W / 2$ (target is reached) implies a total number of submovements strictly less than $n$. Therefore, $n$ appears as the maximum number of permitted submovements and it would be desirable to let $n \rightarrow+\infty$ to obtain a general formulation of Fitts' law accounting for any number of submovements.

Similarly to the case $n=2$ [Meyer, 1988], one could argue that when $D / W$ is large (hence $T$ is large) (11) can be approximated by its highest-degree term:

$$
\frac{D}{W}=\frac{1}{2} E_{n}(2 T) \approx \frac{1}{2} \frac{(2 T)^{n}}{n !}
$$

so that (12) is indeed approximated by an $n$th root law:

$$
T=\frac{1}{2} \sqrt[n]{n ! \frac{D}{W}} .
$$

However, this is not a genuine power model since as $n \rightarrow+\infty$, the multiplying slope factor explodes: $\sqrt[n]{n !} \sim n / e \rightarrow+\infty$ (see Figure 6$)$.

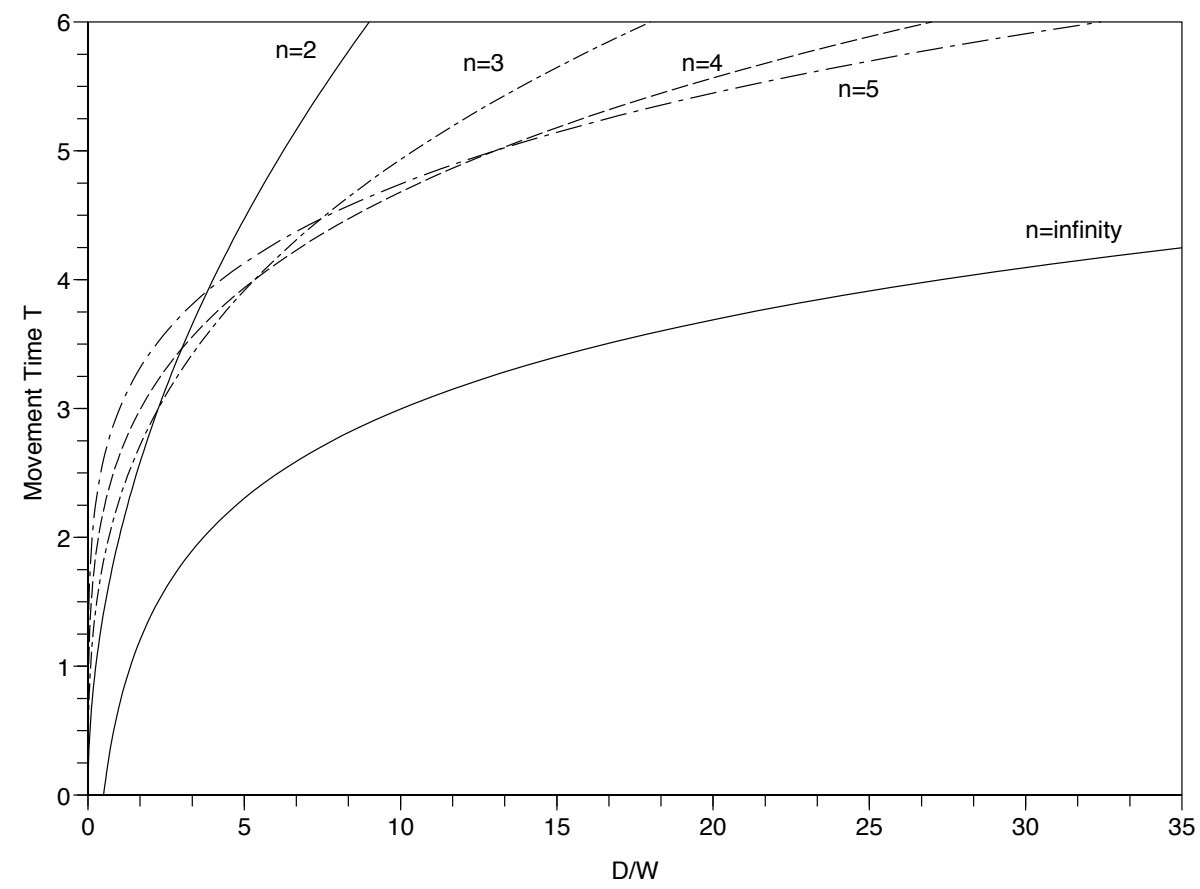

FIGURE 6. Approximated $n$ th-root laws (14) for increasing values of $n$.

In contrast, as Smith [1988] noticed, for any value of $D / W$ (including small ones), the partial sum (11) rapidly converges to the exponential as $n \rightarrow+\infty$ :

$$
\frac{D}{W}=\frac{1}{2} E_{n}(2 T) \longrightarrow \frac{1}{2} \exp (2 T),
$$

and so the final result is logarithmic (see Figure 7):

$$
T=\frac{1}{2} \log _{e}\left(2 \frac{D}{W}\right) .
$$


Comparison of Figures 6 and 7 shows that even for particular moderate values of $n$, the $n$ th-root power function does not approximate the stochastic optimizedsubmovement model's predicted movement times. The exact solution (12) is rather quasi-logarithmic and rapidly converges to the logarithmic law (16).

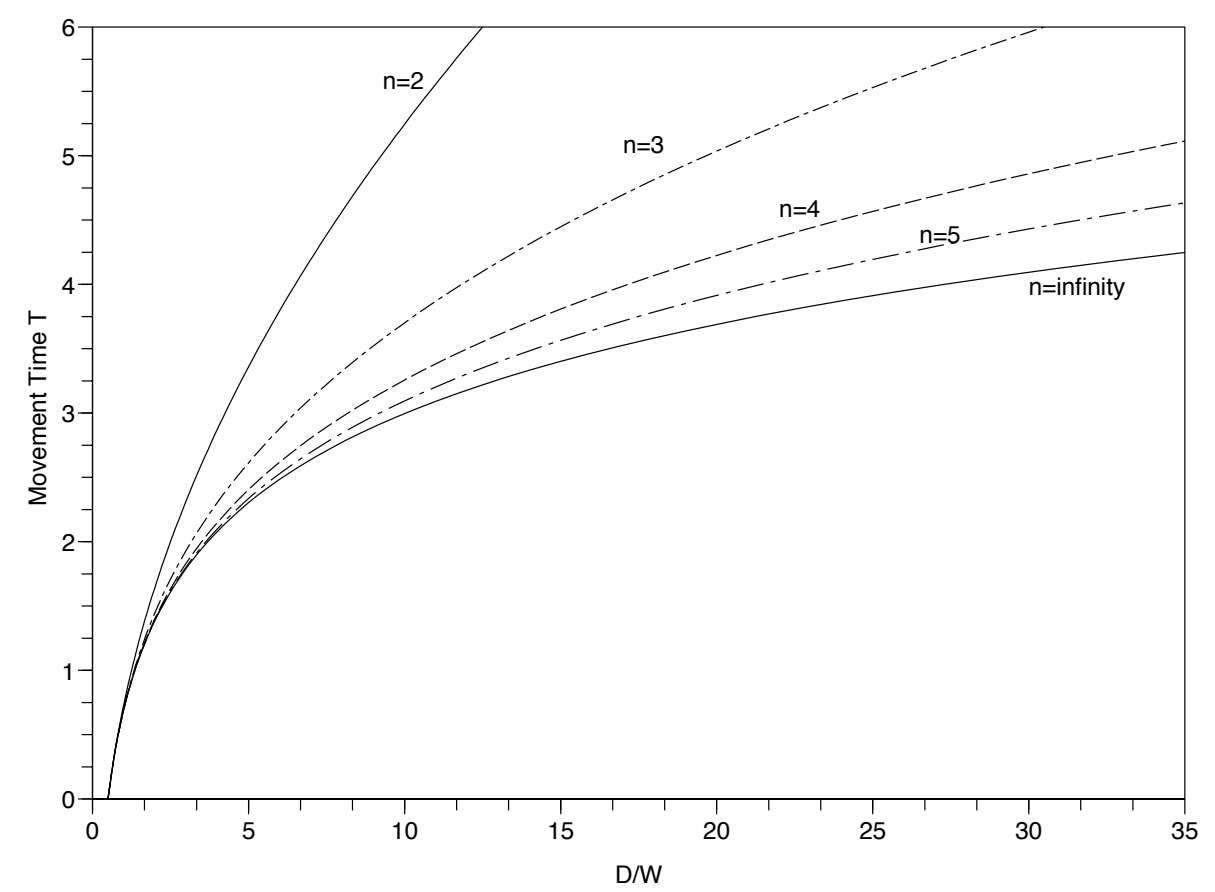

FIGURE 7. Exact laws (12) rapidly converging to the logarithmic law (16) as $n \rightarrow \infty$.

\section{CONCLUSION}

Our mathematical analysis shows that for multiple submovements, the solution of the stochastic optimized-submovement model cannot be identified with a power law of the form (1e), even to a rough approximation, as the number $n$ of submovements grows large. Not only does the power model fail to encompass the logarithmic model, the submovement theory does not yield a genuine power law; it is, rather, a quasi-logarithmic law (12), which rapidly converges to a logarithmic law of the form (1a) or (16).

Since Meyer et al.'s stochastic optimized submovement theory [1988, 1990] is generally considered the best explanation of Fitts' law to date, the community should be aware that the supposedly resulting power model is in fact a logarithmic model reminiscent of Fitt's original formulation - as was incidentally the case for the older deterministic iterative-corrections model.

In addition, our rigorous analysis shows that the two classes of candidate mathematical descriptions of Fitts' law are not equivalent and this, we feel, should stimulate experimental research on the subject.

Acknowledgement. The authors would like to thank the anonymous reviewers for their constructive suggestions. We are indebted to one of them for pointing out references [Kvålseth, 1980, 1993]. 


\section{REFERENCES}

CROSSMAn E.R.F.W., GOOdeVE P.J. (1963), "Feedback control of hand-movement and Fitts' law", Proc. Exp. Psychology Society, Oxford (England).

FITTS P.M. (1954), "The information capacity of the human motor system in controlling the amplitude of movement", J. Exp. Psychology 47, pp. 381-391.

FITTS P.M., PETERSON J.R. (1964), "Information capacity of discrete motor responses", J. Exp. Psychology 67, pp. 103-112.

GUiARD Y., Bourgeois F., MOtTet D., BEAUdouin-lafon M. (2001), "Beyond the 10-bit barrier: Fitts' law in multi-scale electronic worlds", Proc. Interaction HommeMachine / Human-Computer Interaction (IHM-HCI 2001), Lille (France), in A. Blandford, J. Vanderdonckt, and P. Gray (eds.), People and Computers XV - Interactions without frontiers, London, Springer, pp. 573-587.

GUIARD Y., OLAFSDOTTIR H.B. (2011), "On the measurement of movement difficulty in the standard approach to Fitts' law", PLoS ONE 6(10),

http://dx.doi.org/10.1371\%2Fjournal.pone.0024389.

KVÅlseth T.O. (1980), "An alternative to Fitts' law," Bull. Psychonomic Soc. 16(5), pp. 371-373.

KVÅLSETH T.O. (1993), "The paternity of the power law of human motor control," Perceptual 83 Motor Skills 76, pp. 277-278.

MACKENZIE I.S. (1989), "A note on the information-theoretic basis for Fitts' law," J. Motor Behavior 21, pp. 323-330.

MEYER D.E., ABRAMS R.A., KORNBLUM S., WRIGHT C.E., SMITH J.E.K. (1988), "Optimality in human motor performance: Ideal control of rapid aimed movements", Psychological Review 95(3), pp. 340-370.

MEYER D.E., SMith J.E.K., KORNBlum S., ABRAMS R.A., WRIGHT C.E. (1990), "Speedaccuracy trade-offs in aimed movements: Toward a theory of rapid voluntary action", in M. Jeannerod (ed.), Attention and performance XIII, Hillsdale (NJ), Erlbaum, pp. 173-226.

PLAMONDON R., ALIMI A.M. (1997), "Speed/accuracy trade-offs in target-directed movements," Behav. Brain Sciences 20, pp. 279-349.

PLATT J.R. (1964), "Strong inference", Science 146(3642), pp. 347-353.

RIOUL O., GUIARD Y. (to appear), "The power model of Fitts' law does not encompass the logarithmic model", Proc. 2011 Meeting of the European Mathematical Psychology Group (EMPG 2011), Paris, August 29-31, Electronic Notes in Discrete Mathematics.

SHANnON C.E. (1948), "A mathematical theory of communication," Bell Syst. Tech. J. 27, pp. 623-656.

SMITH J.E.K. (1988), "Rapid aimed movements and the speed-accuracy trade off: Optimal strategies", C. Coombs Memorial Symp., Ann Arbor, in Brown, D.R. and Smith, J.E.K. (eds.), Frontiers of mathematical psychology: Essays in honor of Clyde Coombs, New York, Springer, pp. 193-202.

WELFORD A.T. (1960), "The measurement of sensory-motor performance: Survey and reappraisal of twelve years progress", Ergonomics 3, pp. 189-230.

Wobbrock J.O., CUTREll E., HARAdA S., MACKENZIE I.S. (2008), "An error model for pointing based on Fitts' law", Proc. CHI'2008, New York, ACM Press, pp. 1613-1622.

WOODWORTH R.S. (1899), "The accuracy of voluntary movement", Psychological Review 3(13) pp. 1-114. 